Revue internationale P.M.E.

Économie et gestion de la petite et moyenne entreprise

\title{
La Gestion des Ressources Humaines en PME : quelques repères pour la recherche...
}

\section{Odile Uzan}

Volume 3, numéro 1, 1990

URI : https://id.erudit.org/iderudit/1007949ar

DOI : https://doi.org/10.7202/1007949ar

Aller au sommaire du numéro

Éditeur(s)

Presses de l’Université du Québec

ISSN

0776-5436 (imprimé)

1918-9699 (numérique)

Découvrir la revue

\section{Citer cette note}

Uzan, O. (1990). La Gestion des Ressources Humaines en PME : quelques repères pour la recherche... Revue internationale P.M.E., 3(1), 105-116.

https://doi.org/10.7202/1007949ar d'utilisation que vous pouvez consulter en ligne.

https://apropos.erudit.org/fr/usagers/politique-dutilisation/ 


\section{La Gestion des Ressources Humaines en PME : quelques repères pour la recherche...}

Odile UZAN

ERFI, Université de Montpellier III

Les recherches relatives à la gestion des ressources humaines (G.R.H.) sont rares et relativement espacées. En France, seules quelques recherches régionales sont répertoriées à ce jour (Mahé de Boislandelle, 1988).

Cette note de lecture a pour objet d'apporter une contribution à la connaissance des recherches en GRH dans les PME au travers notamment d'une recension des publications américaines au cours de la dernière décennie.

L'exploration n'est certes pas exhaustive; la bibliographie retenue demeure somme toute limitée et difficile à établir; elle reflète les thèmes les plus souvent abordés : les pratiques des PME au niveau global de la GRH, les politiques de rémunérations et les politiques de «maintenance» des ressources humaines.

\section{Les pratiques de GRH...}

\section{$1.1 \quad$...la problématique}

Au début des années 1980, la GRH en PME a fait l'objet d'un double regain d'intérêt aux Etats-Unis. Le premier, relatif à la GRH, traduit les revendications croissantes des salariés en matière de «qualité de vie au travail» ainsi que l'accrois-

- Odile Uzan est maître de conférence à l'Université Paul-Valéry (Université de Montpellier lli) et membre de l'ERFI. Elle coordonne aussi les travaux du groupe d'études en organisation des ressources humaines. Adresse : Université Paul-Valéry, B.P. 5043, 34032 Montpellicr Cedex, tél. : 67.14.20.00 
sement des contraintes légales et des orientations gouvernementales dans ce domaine. Le second, relatif à la PME : par son rôle dans l'économie nationale américaine, elle oblige les différents acteurs à s'interroger sur ses facteurs de succès et d'échec. A l'origine de la popularisation de ce regain d'intérêt, se situe la presse généraliste et spécialisée.

Les sondages réalisés ont tous souligné le fait que les problèmes liés au personnel arrivent en tête des facteurs d'échecs. Ces problèmes s'expriment le plus souvent comme la «difficulté de trouver des employés compétents et de les motiver pour les rendre performants».

Cette médiatisation a eu pour résultat de générer de nombreux articles normatifs recommandant aux PME «comment faire mieux» tout en renforçant l'absence notoire d'articles pragmatiques analysant les pratiques réelles des entreprises.

Tous les travaux de recherche se définissent dans ce contexte comme une contribution à la connaissance des pratiques de GRH en PME destinée à fournir une base rigoureuse aux recommandations qui peuvent être faites dans ce domaine.

\section{$1.2 \quad$...les résultats}

\section{Des résultats qui confirment l'état des connaissances} acquises à ce jour

La PME américaine, selon l'enquête de Mac Evoy (1984), se caractérise par une faible planification des besoins notamment en matière de recrutement et de promotion; dans le meilleur des cas, la prévision se fait à moins d'un mois. Les procédures de recrutement sont peu créatives, souvent limitées aux annonces dans la presse et aux candidatures spontanées; elles n'utilisent jamais les méthodes de promotion et de validation. La formation est le plus souvent (84\% des entreprises) limitée à la formaiton sur le tas.

Dans le domaine de la satisfaction au travail, si 70\% des entreprises pensent que leurs employés sont satisfaits, la plupart subissent une rotation sur embauche nouvelle élevée (entre 37 et 53\% dans les 2 premières années).

La rotation du personnel devient un indicateur de performance très significatif au niveau de la PME. L'étude de Rocha Jr et Khan (1985) indique que 50\% des actions d'améliorations de GRH mises en oeuvre par les PME ont eu un impact positif sur la rotation du personnel et nul sur l'absentéisme.

L'hypothèse selon laquelle :

- les comportements productifs de l'homme au travail varient selon la taille de l'organisation

- ces comportements ne peuvent être évalués par les mêmes indicateurs dans la GE, la PME et la PE se trouve supportée par ce type de résultats.

Si dans la GE, l'absentéisme est un indicateur significatif du rejet de la 
situation de travail et un pré-indicateur du départ potentiel du travailleur, il serait moins performant dans la PME et la PE et ce, au profit de l'indicateur rotation.

Dans une organisation à taille réduite, la pression sociale du groupe de travail sur l'individu est forte et peut dissuader les comportements déviants tel qu'un absentéisme jugé immodéré par le groupe.

Le salarié mécontent de sa situation de travail et qui ne trouverait pas de modes adéquats d'expression refoulera dans un premier temps son insatisfaction puis finira par quitter l'entreprise.

Ainsi la proximité humaine et la prétendue convivialité interne de la PME ne joueraient favorablement que pour l'information descendante. L'effet serait moindre pour l'information ascendante et le problème de l'expression des salariés se poserait au fond avec la même acuité dans la GE, la PME et la PE.

Les analyses demandent à être confirmées; si l'hypothèse de la subrogation de l'absentéisme par la rotation est vérifiée, alors la rotation du personnel pourrait apparaître comme une faiblesse spécifique de la PME par rapport à la GE, en ce sens que la PME pourrait se trouver confrontée à un comportement visible binaire de type «je reste ou je pars».

Compte tenu de ce que nous savons du coût de la rotation subie par la GE, il paraît important d'établir la liste opératoire des dysfonctionnements liés au rejet de la situation de travail et des indicateurs susceptibles d'évaluation socioéconomique à retenir dans le cadre du pilotage social de la PME.

Par ailleurs, on peut formuler l'hypothèse que plus que pour la GE, où la pression sociale du groupe sur l'individu est à certains égards plus diluée, la PME doit imaginer des modalités spécifiques d'expression des travailleurs susceptibles d'éviter le «refoulement» du mécontentement et le départ brutal du salarié.

Dans le cadre d'un espace sociologique interne finalement plus contraignant, de ressources financières limitées et d'une fragilité par rapport à l'environnement externe élevée, un effort de créativité en matière de GRH dans le domaine de l'expression devrait donc être réalisé.

Tab. 1 Impacts des améliorations en GRH dans l'entreprise

\begin{tabular}{|c|c|c|c|}
\hline \multicolumn{2}{|c|}{$\begin{array}{l}\text { Indicateurs } \\
\text { financiers }\end{array}$} & $\begin{array}{l}\text { Indicateurs } \\
\text { d'exploitation }\end{array}$ & $\begin{array}{c}\text { Indicateurs } \\
\text { socio-économiques }\end{array}$ \\
\hline $\begin{array}{l}\text { Ventes : } \\
\text { Profits : } \\
\text { Cash flow : } \\
\text { ROL : } 13 \%\end{array}$ & $\begin{array}{l}50 \% \\
50 \% \\
50 \%\end{array}$ & $\begin{array}{l}\text { Qualité produits/services : } 38 \% \\
\text { Inventaire/stocks : } 0 \%\end{array}$ & $\begin{array}{l}\text { Absentéisme : } 0 \% \\
\text { Rotation : } 50 \% \\
\text { Productivité : } 63 \%\end{array}$ \\
\hline
\end{tabular}

- Modes le lecture : $50 \%$ des actions d'amélioration dans le domaine de la GRH ont eu un impact positif sur les ventes, le profit, etc.

Source : Rocha et Khan (1985) 
Mais l'intérêt de l'étude de Rocha Jr et Khan ne se limite pas à la rotation; l'impact des actions d'améliorations en matière de GRH en PME serait en effet positif sur un nombre important d'indicateurs de performance de l'entreprise.

\section{Quelques questions face à ces résultats :}

- quel est le mode d'évaluation utilisé? La relation biunivoque de cause à effet, entre GRH et indicateurs financiers notamment, est troublante. Dans ce type de logique fort contestable à notre sens, en quoi et comment a-t-on vérifié que les choses étaient alors égales par ailleurs?

Peut-être convient-il de préciser que le mode d'évaluation est ici moins important à souligner que la perception qu'ont les dirigeants de l'impact des actions de GRH sur la performance globale de la PME?

Dans ce cas, il faudrait quand même poursuivre les investigations pour comprendre pourquoi malgré cette conscience du rôle joué par la GRH, les PME «investissent» souvent tard et mal dans ce domaine...

- quel peut-être le modèle de régression sous-jacent de l'impact de la GRH sur la performance globale de la PME? Comme le souligne les auteurs, en considérant que la plupart des entreprises en compte n'ont ni un service «Personnel» ni un programme explicite en GRH, l'impact des actions entreprises peut être d'autant plus important qu'il se compare à un niveau zéro.

- Au fur et à mesure de l'apprentissage organisationnel dans le cadre du cycle de vie de l'entreprise, comment l'impact des actions de GRH sur la performance globale de la PME risque-t-il d'évoluer?

\section{Des résultats qui surprennent...}

Toujours selon Mac Evoy, les PME américaines, au delà du seuil d'effectifs de 86 salariés, structurent de façon indépendante un Service du Personnel, composé en moyenne de 4 personnes. Bien que Mac Evoy a la prudence de préciser que le nombre de personnes le plus fréquent est 1 , le chiffre de 4 surprend et appelle une approche plus fine de ce résultat.

Bien plus étonnant est le fait révélé par l'enquête selon laquelle $77 \%$ des entreprises développent des procédures écrites de politiques du personnel à l'exception des définitions de fonctions rapidement esquissées au moment du recrutement, comme l'exige d'ailleurs la réglementation américaine.

Un tel résultat contredit de façon sensible toutes les hypothèses et les observations réalisées à ce jour, notamment au niveau français. Une explication du résultat selon la taille, la nature de la politique de la GRH et la forme que revêt la procédure écrite aurait été fort utile.

Autre fait étonnant :71\% des PME américaines disposent d'une procédure formelle d'évaluation des performances à partir d'une grille de «mesures objectives 
de productivité». Ces évaluations sont faites au cours d'entretiens annuels où sont abordés le plus souvent de façon simultanée les aspects de performance et de rémunération. Bien que Mac Evoy critique le fait d'évaluer dans la même réunion la performance et la rémunération, $88 \%$ des responsables de PME estiment leur dispositif d'évaluation de la performance satisfaisant alors que $10 \%$ seulement des dirigeants de GE s'en satisfont.

Cet écart de satisfaction serait imputable selon Mac Evoy à la capacité des PME à pouvoir mesurer objectivement la performance au travail; la définition des emplois et du rendement des travailleurs affectés à ces emplois serait plus facile que dans la GE et pourrait constituer une force spécifique de la PME par rapport à la GE; la PME subirait une moindre dilution des performances des travailleurs et de l'évaluation de ces performances. En tout état de cause, le pourcentage de $77 \%$ paraît fort élevé par rapport aux hypothèses et observations françaises.

La recherche serait à approfondir dans deux directions :

- comment se comportent les PME françaises par rapport à l'évaluation du personnel?

- quelles sont les difficultés méthodologiques liées à l'évaluation de la productivité du personnel et celles spécifiques aux PME?

\section{Les rémunérations dans les PME...}

La rémunération est le thème le plus repris par la littérature anglo-saxonne dans le domaine de la GRH en PME (Amba-Rao et Pendse, 1985).

On parlera ici de politiques de «compensation» pour désigner les options conçues en contrepartie de la contribution de l'employé à la firme.

Notons la subtile différence sémantique; le terme américain «compenser» trouverait une juste traduction dans «équilibrer», «dédommager», «réguler», avec l'idée implicite que le travailleur subirait une forme de préjudice ou une dysfonction qui obligerait au dédommagement et/ou à la régulation.

Le terme de politique de «rémunérations» utilisé en France pour désigner les mêmes séries d'options relatives aux salaires et aux avantages complémentaires, trouverait un juste synonyme dans politiques de «rétribution», qui renvoie à l'obligation sociale de payer pour un travail fourni.

La «compensation» renverrait à la notion de justice sociale alors que la «rémunération» renverrait à celle de droit du travail...

L'objet des recherches est de connaître quels sont les démarches et les outils utilisés pour le calcul des salaires et quels sont les avantages complémentaires offerts par les PME? 


\subsection{Des résultats communs...}

Dans l'esprit de dégager des tendances spécifiques aux PME, voire même des lois de comportement des PME en matière de GRH, attardons-nous d'abord sur les résultats communs aux différentes études passées en revue.

En ce qui concerne la méthodologie d'élaboration des salaires, il semblerait que 30\% des PME américaines utilisent les résultats d'enquêtes sur les salaires de la profession ou de l'industrie considérée pour déterminer les bases de calcul des rémunérations. Ce chiffre indique que les dirigeants de PME ont bien le souci de leur environnement social et la volonté de chercher à se positionner stratégiquement par rapport à cet environnement; ce chiffre reste cependant faible. Comment expliquer que $70 \%$ des dirigeants de PME déterminent la base de calcul des salaires sans référence explicite aux données du marché? Amba Rao et Pendse avancent à ce niveau 3 hypothèses explicatives :

- méconnaissance par la majorité des PME de l'existence de telles enquêtes,

- incapacité de fait à les utiliser (données inadaptées, manque de compétences internes pour les adapter au moindre coût...),

- absence de besoins du fait de la taille et/ou de l'état pléthorique de l'offre en matière de qualifications par rapport à la demande.

En ce qui concerne les avantages complémentaires, on retrouve comme point commun aux différentes études :

- une même typologie par nature d'avantages,

- un poids identique dans les pratiques managériales.

Les avantages complémentaires se décomposent en effet selon les catégories ordonnées suivantes :

- vacances et repos payés (70\% des PME font bénéficier leur personnel de ce type d'avantages),

- assurances-vie et santé (60\%),

- fonds et plans de retraite (35\%),

- indemnités de licenciement (15\%).

Il n'existe pas de couverture sociale collective aux USA et les assurances contractées par le biais de l'employeur constituent le plus souvent la seule couverture du salarié pour la prise en charge de ses problèmes de santé et de ceux de sa famille. Ce qui est considéré comme un avantage complémentaire aux USA a, au fond, une importance vitale pour le salarié et sans doute un impact sur le comportement productif beaucoup plus important qu'en France où l'avantage existe sous forme de mutuelle réellement complémentaire à la couverture de base assurée par la Sécurité Sociale. 


\section{Des résultats contradictoires...}

Les méthodes d'analyse de l'emploi pour déterminer la base de rémunération pour chacun des emplois existants dans la firme sont trop opposés dans les principales enquêtes : selon l'enquête de Mac Evoy, 1\% des PME réaliserait ce type d'analyse pour $40 \%$ pour l'enquête de Amba Rao et Pendse. Or ce type de pratique témoigne du souci qu'ont les dirigeants de l'équité de la structure interne des rémunérations et de la valorisation de cette équité auprès des différentes catégories internes.

De la même façon, les résultats relatifs aux pratiques des PME en matière de rémunérations par objectifs sont trop opposés pour être retenus ( $2 \%$ dans l'enquête de Mac Evoy et 20\% pour celle de Amba Rao et Pendse). Enfin, 39\% des PME de l'échantillon retenu par Ambo Rao et Pendse utilisent des échelles de salaires plutôt que des taux de rémunérations uniques.

Il serait intéressant de vérifier si les $40 \%$ de dirigeants qui reconnaissent l'intérêt d'une rémunération différenciée selon le type d'emploi sont les mêmes $40 \%$ qui réalisent effectivement une analyse des emplois internes...

Au plan méthodologique, on notera que l'échantillon d'Amba Rao et Pendse est composé à plus de $80 \%$ de PME dans le secteur des services; ce qui pourrait conduire à penser au vu de certains résultats que les pratiques en matières de GRH dans ce secteur seraient plus évoluées que celles du secteur industriel... cela induit la nécessité de prendre en compte la différenciation par secteurs dans les travaux exploratoires en cours et à venir.

Pour conclure sur les aspects de rémunérations, nous ferons référence à une étude originale réalisée par PE Cooley et CE Edwards(1985). L'âge du «capitaine» a-t-il une influence sur les politiques de rémunérations dans les PME? Oui, semblent répondre les 2 chercheurs qui, après une démonstration quelque peu laborieuse utilisant les modèles de régression, proposent quelques résultats à approfondir :

- les pratiques de rémunérations sont liées aux droits de propriété du dirigeant de la PME,

- la variable d'âge du dirigeant est corrélée de façon significative avec la variable d'ancienneté des droits de propriété du dirigeant,

- la rémunération du dirigeant semble varier avec son âge selon une logique économique qui tend à minimiser les obligations fiscales en jouant alternativement sur lc niveau des profits de l'entreprise et sur les revenus des dirigeants,

- l'attitude du propriétaire dirigeantà l'égard de sa propre rémunération aurait une influence sur la politique de rémunération des salariés de la PME.

Ainsi par exemple, les dirigeants, qui en vieillissant tendraient à minimiser leur niveau de rémunération, pourraient par là même sous évaluer le niveau des salaires requis pour attirer et retenir des cadres professionnels dans la PME. 


\section{Les pratiques de «maintenance»...}

Les pratiques de «maintenance» sont les options destinées à retenir les salariés dans la firme par la satisfaction des attentes relatives à leur situation de travail. Les différentes options sont rassemblées dans une typologie incluant l'intégration, la communication et la participation, la négociation et la discipline, les services rendus aux salariés.

\section{1 -...'intégration}

Les études menées dans le cadre des GE ont démontré l'importance socioéconomique de l'intégration au niveau de la productivité et de la rotation, notamment de la rotation sur embauches nouvelles. Selon l'étude d'Amba Rao et Pendse, seulement $23 \%$ des entreprises ont des procédures d'intégration écrites et détaillées. Un peu plus de $67 \%$ des entreprises affirment cependant disposer d'un programme informel de soutien à l'intégration des nouveaux salariés fondé sur la connaissance du poste de travail, la présentation des collègues immédiats et l'initiation aux aspects organisationnels généraux de l'entreprise...

\section{2 ...la communication et la participation}

La plupart des entreprises interrogées considèrent que la participation des salariés par la formulation de suggestions relatives :

- à l'amélioration de l'environnement du travail,

- à la fixation d'un objectif de résultat,

- à la conception du travail,

est utile pour introduire des changements et/ou améliorer la satisfaction des salariés. Cependant, moins de 50\% de ces entreprises utilisent réellement une approche de suggestions des employés. Si le résultat est encourageant, il mérite un approfondissement sérieux pour connaître les enjeux, les impacts des politiques mises en oeuvre et les limites inhérentes aux outils utilisés.

\section{3 ...la négociation et la discipline}

L'efficacité des politiques de négociation et de résolution des conflits dépend en général du niveau de formalisation retenue, de la connaissance des dispositifs par les salariés et de leur plus ou moins grande adhésion à ces dispositifs. Si 80\% des PME indiquent l'existence d'un dispositif, seulement $30 \%$ ont une procédure véritablement définie et $25 \%$ annoncent que le dispositif est connu par les salariés; cependant $20 \%$ seulement des procédures de traitement sont écrites.

On retrouve pratiquement les mêmes pourcentages pour les dispositifs disciplinaires : $80 \%$ disent qu'ils existent, $30 \%$ que ces dispositifs sont définis selon 
une liste des actions disciplinaires, $30 \%$ que cette liste est connue des salariés et $25 \%$ que cette liste est écrite... La distorsion qui existe entre les croyances et les représentations des dirigeants de PME et les efforts effectivement mis en oeuvre pour expliciter et communiquer selon un mode managérial les schémas et les politiques qu'ils ont en tête est tout à fait remarquable. Les dirigeants ont-ils conscience de cet écart et cherchent-ils inconsciemment ou non à le maintenir pour préserver leur zone de pouvoir (flou sur les domaines et les frontières), leur autorité personnelle (celui qui sait) ou leur flexibilité organisationnelle (modifications des règles selon les cas de figure)? Cet état de fait induit-il des insatisfactions explicites susceptibles d'être reliés à des indicateurs de dysfonctionnement dont l'évaluation socioéconomique permettrait de vérifier l'ampleur de la contre performance éventuelle que subirait la PME du fait de ses pratiques en GRH?

\section{Conclusion}

Les travaux relatifs à la GRH en PME restent trop rares pour que l'on puisse à ce jour établir une modélisation suffisamment exhaustive et opératoire. Un tableau récapitulatif (le tableau 2 présenté en annexe) des principales caractéristiques de la GRH en PME peut cependant commencer à être établi. Un début de comparaison thématique avec la GE semble supporter l'hypothèse selon laquelle :

- les domaines de la GRH seraient identiques quelle que soit la taille des entreprises,

- les modes de fonctionnement et de développement de ces domaines seraient différents du fait des spécificités de la PME; ce sont ces différences qu'il appartient au chercheur de déceler.

La recherche interroge cependant au plan méthodologique. Il devient banal de dire que les PME se caractérisent par un degré élevé d'hétérogéniéité. La plupart des recherches menées à ce jour dans le cadre de la PME utilise la méthodologie de l'enquête et fonde leurs résultats sur des populations réduites (entre 20 et 60 entreprises) (le tableau 3 en annexe présente la méthodologie d'enquête des études analysées).

Pourquoi le choix quasi unanime de la méthodologie de l'enquête? Cet outil, fondé implicitement sur le principe de standardisation des entreprises, ne convientil pas mieux de ce fait aux recherches effectuées dans le cadre des GE? Il paraît en tout état de cause difficile de vouloir trouver des faits nouveaux et différents avec des outils anciens... Par ailleurs l'enquête est-elle la meilleure méthode pour saisir l'écart entre les représentations du dirigeant sur le fonctionnement de la PME et la réalité de ce fonctionnement? La question du mode exploratoire reste posée pour approfondir les premiers résultats collectés à ce jour en matière de GRH dans la PME.

Enfin, il paraît surprenant de voir les chercheurs se satisfaire de la faiblesse de l'échantillon. Ilsconsidèrent sans doute au regard de l'hétérogénéité des PME, que 
l'on ne peut trouver des résultats significatifs qu'en atomisant suffisamment le domaine exploré. Cela paraît plausible mais pose alors le problème de lacomparaison entre les micro populations analysées... On peut cependant émettre l'hypothèse que la méthodologie par enquête sur des populations réduites produit presque autant de résultats consensuels que de résultats contradictoires; pour accélérer la recherche et éviter d'aboutir à des impasses, peut-être faudrait-il alors encourager les investigations menées sur des populations plus nombreuses, à partir d'un découpage au moins régional, voire national.

Tab. 2 Pratiques de GRH dans les PME et les GE

\begin{tabular}{|c|c|c|c|}
\hline & GRH & PME & GE \\
\hline 0 & Intérêt pour la GRH & Faible & En fort développement \\
\hline 0 & $\begin{array}{l}\text { Intérêt pour le type } \\
\text { d'entreprise }\end{array}$ & en développement & fort \\
\hline 0 & $\begin{array}{l}\text { Planification des } \\
\text { besoins }\end{array}$ & $\begin{array}{l}\text { Faible } \\
\text { (au moins d'1 mois) }\end{array}$ & $\begin{array}{l}\text { Développée en fonction de la taille } \\
\text { et du degré d'internationalisation }\end{array}$ \\
\hline 0 & Recrutement & $\begin{array}{l}\text { Peu créatives } \\
\text { limitées aux annonces } \\
\text { dans la presse et aux } \\
\text { candidatures spontanées }\end{array}$ & $\begin{array}{l}\text { De plus en plus perfectionné; } \\
\text { utilisant des méthodes variées et } \\
\text { faisant appel de plus en plus à des } \\
\text { experts internes et/ou externes }\end{array}$ \\
\hline 0 & Intégration & $\begin{array}{l}\text { Dispositif informel, } \\
\text { à faibles moyens }\end{array}$ & $\begin{array}{l}\text { Moyens matériels et budgets temps } \\
\text { alloués pour faciliter l'intégration }\end{array}$ \\
\hline 0 & Formation & $\begin{array}{l}\text { Limitée à la formation } \\
\text { sur le tas (dans } 84 \% \\
\text { des cas) }\end{array}$ & $\begin{array}{l}\text { Politique de formation en fort } \\
\text { développement tant au plan } \\
\text { quantitatif que qualificatif utilisé } \\
\text { comme un levier fondamental } \\
\text { d'adaptation et de changement }\end{array}$ \\
\hline 0 & $\begin{array}{l}\text { Indicateur de } \\
\text { satisfaction }\end{array}$ & $\begin{array}{l}\text { Rotation du personnel } \\
\text { et notamment rotation } \\
\text { sur embauches nouvelles } \\
\text { (entre } 37 \text { et } 53 \% \text { dans } \\
\text { les } 2 \text { premières années } \\
\text { Productivité (63\%) } \\
\text { Qualité (38\%) }\end{array}$ & $\begin{array}{l}\text { Absentéisme : il reste sous ses } \\
\text { multiples formes l'indicateur } \\
\text { le plus sensible des comportements } \\
\text { productifs } \\
\text { Plus faible dans la GE du fait de la } \\
\text { difficulté d'évaluation } \\
\text { Qualité : plus qu'un indicateur } \\
\text { un véritable projet de mobi- } \\
\text { lisation des RH }\end{array}$ \\
\hline 0 & $\begin{array}{l}\text { Évaluation des } \\
\text { performances }\end{array}$ & $\begin{array}{l}\Delta \text { Grille de «mesures } \\
\text { objectives de productivité» }\end{array}$ & $\begin{array}{l}\text { OFiche d'appréciation globale } \\
\text { des performances }\end{array}$ \\
\hline 0 & Rémunérations & $\begin{array}{l}\text { Faible utilisation des } \\
\text { enquêtes sur la profession } \\
\text { et le secteur }\end{array}$ & $\begin{array}{l}\text { Importance accordée au position- } \\
\text { nement de la firme par rapport au } \\
\text { secteur et aux principaux } \\
\text { concurrents }\end{array}$ \\
\hline 0 & $\begin{array}{l}\text { Participation des } \\
\text { salariés et communi- } \\
\text { cation interne }\end{array}$ & $\begin{array}{l}\text { o Une prise de conscience de } \\
\text { limportance de ces domaines } \\
\text { mais des pratiques réelles } \\
\text { faiblement développées }\end{array}$ & $\begin{array}{l}\text { Des efforts permanents (groupes } \\
\text { d'expression, cercles de qualité; } \\
\text { création de postes spécifiques, etc) }\end{array}$ \\
\hline
\end{tabular}




\begin{tabular}{|c|c|c|c|}
\hline & GRH & PME & GE \\
\hline 0 & $\begin{array}{l}\text { Négociation ef } \\
\text { discipline }\end{array}$ & $\begin{array}{l}\text { En règle générale, on } \\
\text { annonce le quarté suivant } \\
80 \% \text { - existence } \\
30 \% \text { - procédure } \\
25 \% \text { - procédure connue } \\
20 \% \text { - procédure écrite }\end{array}$ & $\begin{array}{l}\text { En général, les procédures } \\
\text { largement écrites et diffusées } \\
\text { intentionnellement pour limiter } \\
\text { les rumeurs et interprétations }\end{array}$ \\
\hline 0 & $\begin{array}{l}\text { Structuration du } \\
\text { service du personnel }\end{array}$ & $\begin{array}{l}\text { - Seuil de structuration } \\
\text { d'un service spécifique : } \\
86 \text { salariés } \\
\text { - Au delà de ce seuil, } \\
\text { composition moyenne: } \\
4 \text { salariés }\end{array}$ & \\
\hline
\end{tabular}

Tab. 3 Description de la méthodologie d'enquête des études analysées

\begin{tabular}{|c|c|c|c|}
\hline & Mac Evoy & Rocha et Khan & Amba Roa et Pendse \\
\hline $\begin{array}{l}\text { Type de } \\
\text { méthodologie }\end{array}$ & $\begin{array}{l}\text { Questionnaire de } 36 \\
\text { items passé par } 1 \text { en- } \\
\text { quêteur, au cours d'1 } \\
\text { entretien de } 30 \mathrm{mn} \text { avec } \\
\text { la personne en charge de } \\
\text { la fonction «Personnel. } \\
\text { après accord de } \\
\text { l'entreprise }\end{array}$ & $\begin{array}{l}\text { Questionnaire standard } \\
\text { s'inscrivant dans une } \\
\text { étude plus large d'obser- } \\
\text { vation sur } 1 \text { période de } 6 \\
\text { ans (1977 à 1982) d'en- } \\
\text { treprises ayant participé } \\
\text { à un programme de } \\
\text { consultation }\end{array}$ & Questionnaire postal \\
\hline $\begin{array}{l}0 \text { Population } \\
\text { de départ }\end{array}$ & $\begin{array}{l}141 \text { entreprises } \\
\text { effectif : } 25 \text { et } 250\end{array}$ & 86 entreprises & 78 entreprises \\
\hline $\begin{array}{l}\checkmark \text { Population } \\
\text { de l'échan- } \\
\text { tillon }\end{array}$ & $\begin{array}{l}54 \text { entreprises } \\
\text { taux de participation } \\
38 \%\end{array}$ & $\begin{array}{l}36 \text { entreprises } \\
\text { taux de participation } \\
42 \%\end{array}$ & $\begin{array}{l}23 \text { entreprises } \\
\text { taux de participation } \\
29 \%\end{array}$ \\
\hline $\begin{array}{l}0 \text { Effectif des } \\
\text { entreprises }\end{array}$ & $\begin{array}{l}38 \% \rightarrow[101 \text { à } 250] \\
62 \% \rightarrow[25 \text { à } 100] \\
\text { effectif }=83 \text { salariés }\end{array}$ & & $<300$ salariés \\
\hline $\begin{array}{l}\Delta \text { Niveau de } \\
\text { syndicalisation }\end{array}$ & $3 \%$ des entreprises & & \\
\hline $\begin{array}{r}\text { Secteurs } \\
\text { d'activité }\end{array}$ & $\begin{aligned} & 78 \% \rightarrow \text { commerce de } \\
& \text { détail } \\
& \text { construction } \\
& \text { industrie } \\
& \text { solde } \rightarrow \text { commerce } \\
& \text { gros } \\
& \text { restauration } \\
& \text { secteur financier } \\
& \text { automobile } \\
&\end{aligned}$ & - éventail assez large & \\
\hline $\begin{array}{l}0 \text { Localisation } \\
\text { géographique }\end{array}$ & $\begin{array}{l}\text { Entreprises relevant } \\
\text { du même code postal } \\
\text { à proximité d'une } \\
\text { métropole dans le } \\
\text { midwest }\end{array}$ & $\begin{array}{l}\text { - Zone limitée, autour } \\
\text { de l'Université de Lowell } \\
\text { dans le Massachussets }\end{array}$ & \\
\hline
\end{tabular}




\section{BIBLIOGRAPHIE}

Cooley, P.L. et Edwards, C.E., (1985), «Age effects on managerial compensation in small firms», American Journal of Small Business, été.

Louart, P., (1983), «PME et logiques sociales», Communication aux journées de recherche en gestion, Lille, janvier.

Mac Evoy, G.M., (1983), «Personnel practices in smaller firms : a survey and recommendations», American Journal of Small Business, $n^{\circ} 2$.

Mac Evoy, G.M., (1984), «Small business personnel practices», American Journal of Small Business, $\mathrm{n}^{\circ} 4$.

Mahé de Boislandelle, H., (1988), Gestion des Ressources Humaines dans les PME, Paris, Economics, $322 \mathrm{p}$.

Marchesnay, M. et Julien,P.A., (1989), La petite entreprise, Paris, Vuibert.

Naro, G., (1986), «Les obstacles à l'embauche dans les PME : du discours aux réalités», ERFI, Montpellier, mai.

Rocha, J.R.J. et Khan, M.R., (1985), «The human resource factor in small business decision making», American Journal of Small Business, automne.

Sacotra, (1984), «Conditions de vie au travail et PME», Cahiers $n^{\circ} 1$ CNRS, Montpellier, février.

Sacotra, (1985-86), «Petites entreprises de services, vie au travail, santé et vie hors travail», Montpellier.

Sita, C., Amba-Rao et Dilip Pendse, (1985), «Human resource compensation and maintenance practices», American Journal of Small Business, automne.

Uzan, O. et alii, (1979), «Manuel de procédure pour l'évaluation des indicateurs socioéconomiques», mai, $40 \mathrm{p}$.

Uzan, O. et alii, (1980), «Évaluation socio-économique comparée de trois modes d'organisation du travail dans une usine de fabrication d'appareils électroménagers», mars, 147p. 